\title{
Quantitative dual-energy CT material decomposition of holmium microspheres: local concentration determination evaluated in phantoms and a rabbit tumor model
}

\author{
Ralf Gutjahr $^{1,2} \cdot$ Robbert C. Bakker ${ }^{3,4} \cdot$ Feiko Tiessens $^{5} \cdot$ Sebastiaan A. van Nimwegen $^{6} \cdot$ Bernhard Schmidt $^{1}$. \\ Johannes Frank Wilhelmus Nijsen ${ }^{5,7}$
}

Received: 29 January 2020 / Revised: 3 June 2020 / Accepted: 20 July 2020 / Published online: 7 August 2020

(C) The Author(s) 2020

\begin{abstract}
Objectives The purpose of this study was to assess the feasibility of dual-energy CT-based material decomposition using dual-X-ray spectra information to determine local concentrations of holmium microspheres in phantoms and in an animal model.

Materials and methods A spectral calibration phantom with a solution containing $10 \mathrm{mg} / \mathrm{mL}$ holmium and various tube settings was scanned using a third-generation dual-energy CT scanner to depict an energy-dependent and material-dependent enhancement vectors. A serial dilution of holmium (microspheres) was quantified by spectral material decomposition and compared with known holmium concentrations. Subsequently, the feasibility of the spectral material decomposition was demonstrated in situ in three euthanized rabbits with injected (radioactive) holmium microspheres.

Results The measured CT values of the holmium solutions scale linearly to all measured concentrations and tube settings $\left(R^{2}=1.00\right)$. Material decomposition based on CT acquisitions using the tube voltage combinations of 80/150 Sn kV or 100/150 Sn kV allow the most accurate quantifications for concentrations down to $0.125 \mathrm{mg} / \mathrm{mL}$ holmium.

Conclusion Dual-energy CT facilitates image-based material decomposition to detect and quantify holmium microspheres in phantoms and rabbits.

Key Points

- Quantification of holmium concentrations based on dual-energy CT is obtained with good accuracy.

- The optimal tube-voltage pairs for quantifying holmium were 80/150 Sn kV and 100/150 Sn kV using a third-generation dualsource CT system.

- Quantification of accumulated holmium facilitates the assessment of local dosimetry for radiation therapies.
\end{abstract}

Keywords Tomography, X-Ray computed $\cdot$ Contrast media $\cdot$ Holmium $\cdot$ Microspheres

\begin{tabular}{|c|c|}
\hline Abbre & tions \\
\hline${ }^{165} \mathrm{Ho}$ & Holmium-165 isotope \\
\hline${ }^{166} \mathrm{Ho}$ & Holmium-166 isotope \\
\hline $\mathrm{CM}$ & Contrast media \\
\hline
\end{tabular}

Ralf Gutjahr and Rob C. Bakker contributed equally to this work.

Johannes Frank Wilhelmus Nijsen

frank.nijsen@radboudumc.nl

1 Computed Tomography, Siemens Healthcare GmbH, Forchheim, Germany

2 CAMP, Technical University of Munich, Munich, Germany

3 Department of Radiology and Nuclear Medicine, University Medical Center Utrecht, Utrecht, The Netherlands

$\begin{array}{ll}\text { CS } & \text { Compton scatter } \\ \text { DECT } & \text { Dual-energy computed tomography } \\ \text { HoMS } & \text { Holmium microspheres } \\ \text { kV } & \text { Kilovolt }\end{array}$

4 Department of Oral and Maxillofacial Surgery, University Medical Center Utrecht, Utrecht, The Netherlands

5 R\&D Imaging \& Software, Quirem Medical BV, Deventer, The Netherlands

6 Department of Clinical Sciences of Companion Animals, Faculty of Veterinary Medicine, Utrecht University, Utrecht, The Netherlands

7 Department of Medical Imaging, Radboudumc, Geert Grooteplein-Zuid 10, 6525 GA Nijmegen, The Netherlands 
PE Photoelectric effect

Sn Tin (filter)

\section{Introduction}

In the 1970s and 1980s, dual-energy CT (DECT) technology demonstrated improved tissue characterization; however, the technique was not widely applied due to limitations like noise in low-kilo voltage $(\mathrm{kV})$ images, acquisition time, and image registration difficulties [1-5]. Nowadays, DECT technology is clinically established as a result of fast technological developments, such as detectors with fully integrated electronics minimizing electronic noise, improved spectral separation using optimized beam pre-filtration, increased scan speed, and improved post processing techniques [6-11].

DECT uses two effective X-ray spectra, either generated by a single $\mathrm{X}$-ray tube switching between two different $\mathrm{X}$-ray spectra (kV-switch CT), by using two separated Xray tubes applying two different voltages (dual-source CT, DSCT), or by resolving an incident spectrum at the scanner (dual-layer CT or photon-counting detector CT). Further image processing enables the quantification of materials by separating their attenuation characteristics into the different contributions of photoelectric absorption and Compton scatter [8, 12-15]. Nowadays, DECT is clinically used for diagnostic purposes such as classification of uric acid versus non-uric acid urinary stones or to quantify contrast media (CM) uptake, e.g., the local concentration of iodine in liver tissue [9, 16-19]. Experimental studies also suggest the use of nonapproved CM with spectral properties that could be utilized for CTA or cancer theranostics.

A particular therapy that potentially would benefit from DECT quantification is selective internal radiation therapy (SIRT) with beta-emitting radioactive holmium-166 microspheres ( ${ }^{166}$ HoMS), which are currently used for radioembolization of liver tumors and intratumoral injection in solid malignancies [20-23]. In vivo dosimetry after therapy application, needed to verify the treatment success, can be performed based on SPECT imaging utilizing the holmium166 gamma radiation or based on magnetic resonance imaging (MRI) utilizing the paramagnetic properties of holmium. Both modalities have shown their possibilities for application in radioembolization therapy; however, for intratumoral therapy, their use might be hampered by resolution limitations or detection limits [23-26].

In intratumoral therapy, high concentrations of microspheres are injected at several locations in the tumor to achieve proper dose levels for the entire tumor. This requires highresolution dosimetry that cannot be achieved by SPECT and quantification of high holmium concentrations which is challenging for MRI.

Conventional CT for quantification of ${ }^{166} \mathrm{HoMS}$, utilizing the high attenuation coefficient of holmium, has previously been explored [24, 25]. CT in general would allow for fast ${ }^{166}$ HoMS quantification of high local concentrations with high spatial resolution and thus accurate local dosimetry at low cost. Although a clear relation was demonstrated between local holmium concentrations and CT signal, discrimination of holmium from, e.g., bone, calcified arteries, or iodinated CM, was found to be difficult [24].

It is expected that DECT can improve the previously identified limitations of conventional CT by utilizing the spectral information that DECT offers combined with the presence of a k-edge at $56 \mathrm{keV}$ that holmium expresses, leading to a sudden increase in X-ray attenuation at that energy.

The objective of this study was to demonstrate the feasibility of DECT-based quantification of non-radioactive HoMS by means of phantom and measurements of rabbit cadavers.

\section{Materials and methods}

In this study, the following experiments were performed: Firstly, a spectral calibration was performed to determine the tube voltage combination-dependent and object size-specific spectral properties of the investigated material. Secondly, contrast media quantification measurements were performed to define detection limits and accuracy. Finally, the feasibility of holmium quantification was tested in a pilot study performed in situ in VX-2 tumor-bearing rabbits. Since only a very small fraction of holmium-165 is converted to holmium166 after neutron activation during production (approximately $0.001 \%$ [23]), no difference is expected in X-ray attenuation between radioactive and non-radioactive microspheres. Therefore, all experiments were performed using ${ }^{165} \mathrm{HoMS}$ to avoid unnecessary radiation risks.

\section{Spectral calibration}

Spectral calibration is required to depict an energy-dependent and material-dependent material vector [12]. For this purpose, a dedicated spectral calibration phantom (QRM GmbH) was scanned using a third-generation dual-energy CT scanner (SOMATOM Force, Siemens Healthcare GmbH). The scans were performed using a dual-energy abdomen protocol; collimation $2 \times 64 \times 0.6 \mathrm{~mm}$ with a flying focal spot in the $\mathrm{z}$ direction, rotation time $0.5 \mathrm{~s} / \mathrm{rot}$, and a pitch of 0.6 . The images were reconstructed using a weighted filtered backprojection algorithm [27] providing linear handling of contrast, noise, and spatial resolution in order to preserve purely quantitative results. All images were reconstructed with a slice thickness of $1.5 \mathrm{~mm}$, an increment of $1.0 \mathrm{~mm}$, a 
quantitative medium smooth reconstruction kernel (Qr44f) used for routine reconstruction of DECT images (50\% value of the modulation transfer function: $\rho_{50}=4.62 \mathrm{lp} / \mathrm{cm}$, no edge enhancement), and a reconstruction field of view (FOV) of $175 \mathrm{~mm}$. The phantom consists of a 10-cm-wide cylinder comprising a synthetic material exhibiting liquid waterequivalent $\mathrm{CT}$ values. Two 2 -cm-diameter syringes (Omnifix Solo, B. Braun) were located in the phantom's center and $3 \mathrm{~cm}$ horizontally offset, respectively. The centric syringe was filled with holmium (III) chloride hexahydrate (Metal Rare Earth Limited (Holmium content 41.3-45.5\% $(\mathrm{w} / \mathrm{w})$ ) suspended in distilled water (Fresenius SE \& Co $\mathrm{KGaA}$ ). Titration of the holmium chloride revealed a $42.0 \%$ holmium content resulting in a final concentration of 10.5 $\mathrm{mg} / \mathrm{mL}$. The known concentration served as a reference to facilitate quantitative measurements. The offset syringe contained pure water as a reference to the phantom's original material. In the spectral calibration, the following effects were investigated using an automatic exposure control (CARE, Dose4D, Siemens Healthcare $\mathrm{GmbH}$ ), the effect of X-ray tube voltage combinations and size-dependent spectral effects (Table 1). Measurements were performed using different Xray tube voltage combinations: $70 / 150 \mathrm{Sn} \mathrm{kV}, 80 / 150 \mathrm{Sn} \mathrm{kV}$, $100 / 150 \mathrm{Sn} \mathrm{kV}$, and $80 / 140 \mathrm{kV}$, whereas $S n$ denotes the utilization of a $0.6-\mathrm{mm}$-thick tin pre-filtration used for increased spectral separation [6]. To investigate sizedependent spectral effects, additional extension rings were used to expand the 10-cm-wide phantom to $15,20,25,30$, or $35 \mathrm{~cm} \mathrm{[7,26,28].}$

\section{Material decomposition algorithm}

The relationship of the material-specific CT values of corresponding spectral $\mathrm{CT}$ acquisitions allows the determination of material vectors unique for the used tube voltages. The used image-based material separation algorithm (syngo.via, VB30A, Siemens Healthcare GmbH) relies on a base transformation and a projection of every measured set of $\mathrm{CT}$ values to preselected orthogonalized and linearly independent base vectors $[8,29]$. In our study, air (- 1000/- $1000 \mathrm{HU}$ for the low-kV and high-kV image, respectively), water $(0 / 0 \mathrm{HU})$ and ${ }^{165} \mathrm{HoMS}$ were selected as base materials. Decomposition into these three materials enables the generation of a material images that constitute local concentrations of ${ }^{165} \mathrm{HoMS}$, as well as virtual non-contrast images, mimicking a native scan where no CM was applied (Fig. 1).

\section{Contrast media quantification measurements}

A serial dilution of HoMS $(0.125-10.0 \mathrm{mg}$ holmium $/ \mathrm{mL})$ was examined to test linearity with various concentrations and the detection limit. The concentration inserts were made of a batch of ${ }^{165} \mathrm{HoMS}$ with a holmium mass percentage of 18.8\% (QuiremSpheres, Quirem Medical B.V.). The microspheres were suspended in an injection solution containing $116 \mathrm{mmol}$ phosphate-buffered saline $(\mathrm{pH} 7.2,77.0 \mathrm{mmol}$ disodium phosphate dibasic dehydrate, Merck Millipore, and 39.0 mmol sodium phosphate monobasic anhydrous, Sigma Aldrich) with polyoxyethylene-polyoxypropylene block copolymer (Pluronic F-68, Sigma-Aldrich, Chemie B.V.) 2\% weight per volume solution. To prevent precipitation of ${ }^{165} \mathrm{HoMS}$ due to their weight $(1.4 \mathrm{~g} / \mathrm{mL})$, an agar solution (MP Agar, Roche Diagnostics) was added. The agar solution was heated to $90^{\circ} \mathrm{C}$ for $10 \mathrm{~min}$, resulting in a transparent fluid. The ${ }^{165}$ HoMS suspension and agar were mixed in a rising concentration of ${ }^{165} \mathrm{HoMS}$ and filled into syringes for the spectral calibration measurements and into $5 \mathrm{-mL}$ Eppendorf tubes for the quantitative comparison of measured and known concentration in the $\mathrm{CT}$ images. Once cooled to room temperature, the agar became solid.

The experimental setup consisted of a 20 -cm-wide (real) water phantom that allowed the positioning of multiple tubes. The solutions were scanned in two groups $(0.125-1.0 \mathrm{mg} / \mathrm{mL}$ and $2.0-10.0 \mathrm{mg} / \mathrm{mL}$ ). The inserts were arranged in an equiangular fashion $5 \mathrm{~cm}$ distant from the phantom's centerline.

In addition to the clinical dose (CARE Dose4D) measurements, a series of high-dose measurements were conducted (Table 2). With the first mode, the accuracy of ${ }^{165} \mathrm{HoMS}$ quantification was assessed under clinical conditions, whereas the maximal tube current allows the determination of a contrast agent in a low noise situation. After the image-based material decomposition, the images were quantitatively assessed and ${ }^{16 \%} \mathrm{HoMS}$ concentrations were compared with the known concentrations.
Table 1 Effective tube currenttime products regarding the different phantom sizes and all tube voltage combinations as investigated in the calibration measurements

\begin{tabular}{lllllll}
\hline Tube voltages (system A/system B) in (kV) & \multicolumn{6}{c}{ Tube current time products for different phantom sizes in (mAs) } \\
\cline { 2 - 7 } & $10 \mathrm{~cm}$ & $15 \mathrm{~cm}$ & $20 \mathrm{~cm}$ & $25 \mathrm{~cm}$ & $30 \mathrm{~cm}$ & $35 \mathrm{~cm}$ \\
\hline $70 / 150 \mathrm{Sn}$ & $16 / 16$ & $18 / 16$ & $37 / 22$ & $80 / 36$ & $180 / 16$ & $410 / 95$ \\
$80 / 150 \mathrm{Sn}$ & $16 / 16$ & $16 / 16$ & $23 / 22$ & $45 / 36$ & $95 / 60$ & $202 / 95$ \\
$100 / 150 \mathrm{Sn}$ & $16 / 16$ & $20 / 16$ & $34 / 22$ & $60 / 36$ & $109 / 60$ & $195 / 95$ \\
$80 / 140$ & $16 / 16$ & $16 / 16$ & $16 / 16$ & $30 / 16$ & $65 / 16$ & $142 / 24$ \\
\hline
\end{tabular}


Fig. 1 Spectral calibration scans are used to generate energydependent and materialdependent material vectors as a prerequisite for the image-based

Therefore, the X-ray absorption of holmium on the applied X-ray spectra was investigated in relation to the size of the object (using $10-35-\mathrm{cm}$ extension rings). Based on two different energy input images, inputs 1 and 2, the material decomposition can be calculated and results in material images constituting of local concentrations of ${ }^{165} \mathrm{HoMS}$, as well as virtual non-contrast images (VNC) material decomposition.

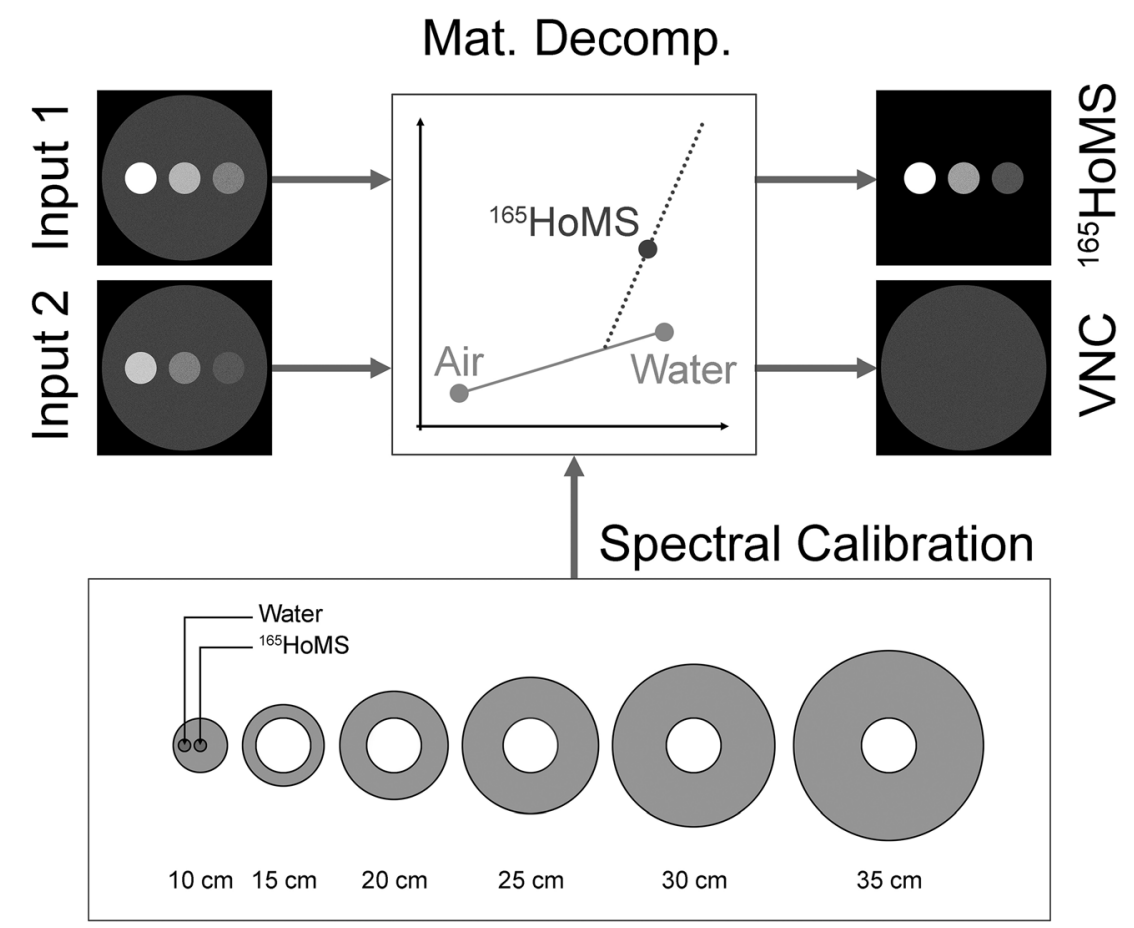

Mat. Decomp.

Equation 1 describes a tolerance term (derived from [30]) discerning as an estimate error for material quantification. The two terms presume the CT value stability and spectral stability to be two independent types of errors. The tolerance scales with increased known concentration $C_{\text {known }}$.

$T=\sqrt{\left(0.5 \frac{\mathrm{mg}}{\mathrm{mL}}\right)^{2}+\left(0.1 \cdot C_{\mathrm{known}}\right)^{2}}$

\section{Animal preparation}

All experiments were performed in conduct with The Netherlands Experiments on Animals Act (1977) and The European Convention for the Protection of Vertebrate Animals used for Experimental Purposes (Strasbourg, 18.III.1986). Approval was obtained from the Utrecht
University Animal Experiments Committee (DEC 2011.III.08.080).

The VX-2 tumor model was described previously [28]. Six tumors were induced by subcutaneous injection of three \pm 1 $\mathrm{mm}^{3}$ viable fragments of VX-2 carcinoma harvested from the donor rabbit. Next, these fragments were injected with $0.1-0.3$ mL PBS into three adult female New Zealand White (NZW) rabbits weighting 3-4 kg. All tumor implantations were performed under analgesia with carprofen $4 \mathrm{mg} / \mathrm{kg}$. During the animal experiments, sedation and analgesia were achieved with a mixture of $0.125 \mathrm{mg} / \mathrm{kg}$ dexdomitor and $15 \mathrm{mg} / \mathrm{kg}$ ketamine.

Subsequently, aliquots with various amounts of ${ }^{166}$ HoMS were administered intratumorally. The exact administered amount, regarding holmium mass (mg), was calculated by measuring the radioactivity of the syringes with ${ }^{166} \mathrm{HoMS}$ before and after injection using a VDC-404 dose calibrator, Veenstra Instruments B.V. Measurements performed on the
Table 2 Overview of the effective current, $\mathrm{Q}_{\mathrm{ref}}$, and radiation dose for all applied tube voltage combinations for the quantification measurements

\begin{tabular}{llccc}
\hline $\begin{array}{l}\text { Tube voltages (system A/ } \\
\text { system B) in }(\mathrm{kV})\end{array}$ & $\begin{array}{l}\text { Tube current } \\
\text { determination }\end{array}$ & $\begin{array}{l}\text { Effective current } \\
\text { in }(\mathrm{mAs})\end{array}$ & $\begin{array}{l}\mathrm{Q}_{\text {ref }} \text { in } \\
(\mathrm{mAs})\end{array}$ & $\begin{array}{l}\text { Dose (CTDI32) in } \\
(\mathrm{mGy})\end{array}$ \\
\hline 70/150 Sn & CARE Dose4D & $212 / 55$ & $380 / 95$ & 4.36 \\
& High Dose & $1083 / 271$ & - & 21.92 \\
80/150 Sn & CARE Dose4D & $105 / 54$ & $190 / 95$ & 3.83 \\
& High Dose & $1083 / 542$ & - & 38.95 \\
$100 / 150 \mathrm{Sn}$ & CARE Dose4D & $60 / 53$ & $190 / 95$ & 4.22 \\
$80 / 140$ & High Dose & $840 / 420$ & - & 48.91 \\
& CARE Dose4D & $73 / 18$ & $132 / 24$ & 3.40 \\
& High Dose & $997 / 181$ & - & 39.24 \\
\hline
\end{tabular}


DECT are primarily based on ${ }^{165} \mathrm{Ho}$ as only a fraction is converted. Animals were sacrificed and preserved in a shielded freezer to let the radioactive holmium decay prior to scanning with the dual-energy CT scanner. The average water equivalent diameter of the rabbit cadaver ranged from 15 to $20 \mathrm{~cm}$, such that the general quantitative detection of the ${ }^{165} \mathrm{HoMS}$ as from the prior phantom scans can be assumed.

\section{Statistical analysis}

After applying the image-based material decomposition algorithm, the quantitative material images were evaluated for ${ }^{165}$ HoMS quantification. Descriptive statistics (mean concentration, $R^{2}$, RMSE, absolute and relative deviation) of the image-based material decomposition measurements were compared with the known concentrations for all tube voltage combinations and dose settings.

\section{Results}

\section{Spectral calibration}

Figure 2 shows that the X-ray absorption of holmium highly depends on the applied X-ray spectra and the size of the object. With an increased diameter, the mean energy of the $\mathrm{X}$-ray spectrum shifts towards higher energies, which, for lighter atoms (presuming no k-edge in the applied X-ray spectrum), results in a decrease of X-ray attenuation and therefore lower $\mathrm{CT}$ values. An increased attenuation and an enhanced $\mathrm{CT}$ value are observed when the mean energy of an X-ray spectrum and its spectral barycenter moves closely beyond the $\mathrm{k}$ edge of a material, where the photoelectric effect becomes dominant. The further the energies shift, the more the measured CT values decrease. For all investigated energies, both

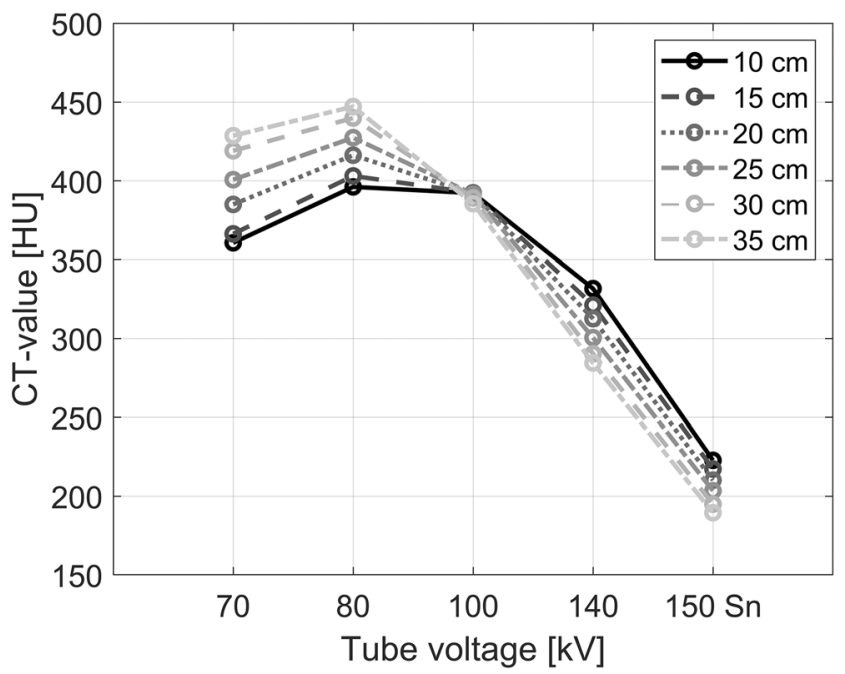

Fig. 2 Measured CT values for $10.0 \mathrm{mg}{ }^{165} \mathrm{HoMS} / \mathrm{mL}$ with regard to different tube voltages and phantom sizes effects, the decreased X-ray attenuation due to increased object size and the simultaneously mean energy shift towards the k-edge of the material (56 keV for Holmium), result in an increase of total X-ray attenuation at 70 and $80 \mathrm{kV}$, but eventually to a decrease in absorption at 100-150 Sn kV (Fig. 2).

\section{Contrast media quantification measurements}

The CT values of the holmium solutions were identical for both dose levels and the image noise level scales square root to the radiation dose reduction (Fig. 3 a and b). Measured concentrations, in the phantom model, using CT quantification were linear (Table 3 ) and slightly lower than the known concentrations (Fig. $3 \mathrm{c}$ and d). Figure $3 \mathrm{e}$ and $\mathrm{f}$ show the increase of deviation with lower holmium concentrations. Given the introduced tolerance term, all quantifications down to 0.125 ${ }^{166} \mathrm{Ho}$-microspheres $\mathrm{mg} / \mathrm{mL}$ were sufficient for all tube voltage combinations and for both clinical dose and high doses. The highest accuracy was observed using $80 / 150 \mathrm{Sn} \mathrm{kV}$, followed by $100 / 150 \mathrm{Sn}, 70 / 150 \mathrm{Sn}$, and $80 / 140 \mathrm{kV}$ tube voltage and high radiation dose.

The measured CT values decreased with lower holmium concentrations, while the noise level remained the same for a tube voltage. This resulted in a factor 3-4 times lower SNR, e.g., from 65.4 to 15.7 for $80 \mathrm{kV}$ with $10 \mathrm{mg} / \mathrm{mL}$ holmium (Fig. 4). The additional value of DECT material decomposition is shown in Fig. 5, illustrating the output of two separate material decompositions, virtual non-contrast images (Fig. 5 a and b) and the quantitatively measured mapping of holmium concentrations (Fig. $5 \mathrm{c}$ and d).

\section{Animal experiment}

Figure 6 depicts two acquired coronary slices of a scanned rabbit cadaver using $80 \mathrm{kV}$ (a), $150 \mathrm{Sn} \mathrm{kV} \mathrm{(b),} \mathrm{and} \mathrm{a} \mathrm{calcu-}$ lated VNC image (c) and a material-specific image (d). The material-specific image segments the holmium and soft tissue to show the exact location of the injected material with respect to the anatomy of the single animals (Fig. 6d). The injected and detected amount of ${ }^{165} \mathrm{HoMS}$ corresponded well with relative deviation ranging between 1 and 11\% (Table 4).

\section{Discussion}

In this article, we presented the first results of DECT-based quantification of holmium microspheres in phantoms and euthanized rabbits.

All measurements showed a linear relation between DECT-based holmium concentrations and actual holmium concentrations. This was observed for all investigated X-ray spectra. In the high-dose acquisitions, the accuracy per measurement is superior due to the reduced noise level compared 


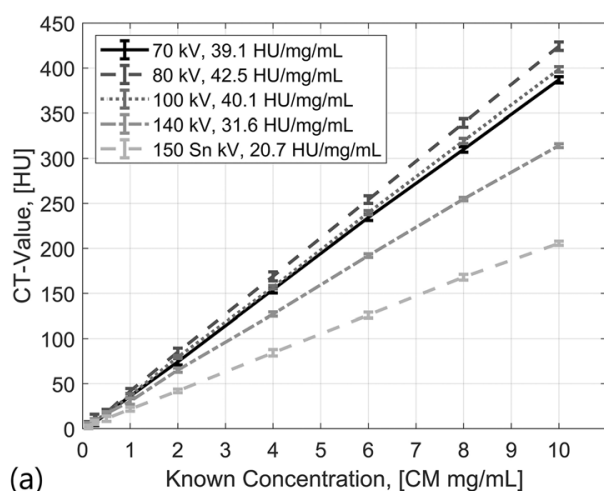

(a)

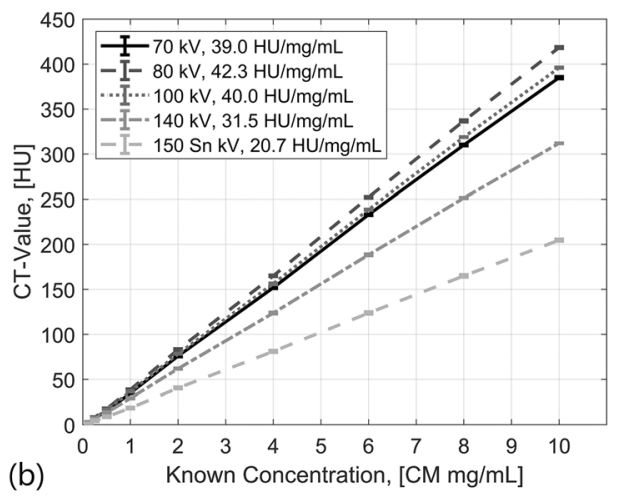

Fig. 3 Slope and CT values for different ${ }^{165} \mathrm{HoMS} \mathrm{mg} / \mathrm{mL}$ solutions in a 20 -cm-wide water phantom. a High-dose measurements, (b) clinical situation with typical clinical radiation dose using automated exposure control. Accuracy of the measured concentrations compared against the known concentrations for high-dose measurements (c) and measurements

with clinical (low) dose acquisitions. In the case of low concentrations of holmium, the reduced CT value can no longer be distinguished from the background signal anymore. This effect propagates through the applied material decomposition algorithm. Ultimately, the quality of the applied material separations based on the image data of the high-dose measurements outperformed the quality of the respective low-dose measurements.

The measured CT values of the ${ }^{165}$ HoMS solutions depended on the investigated phantom sizes. For lower
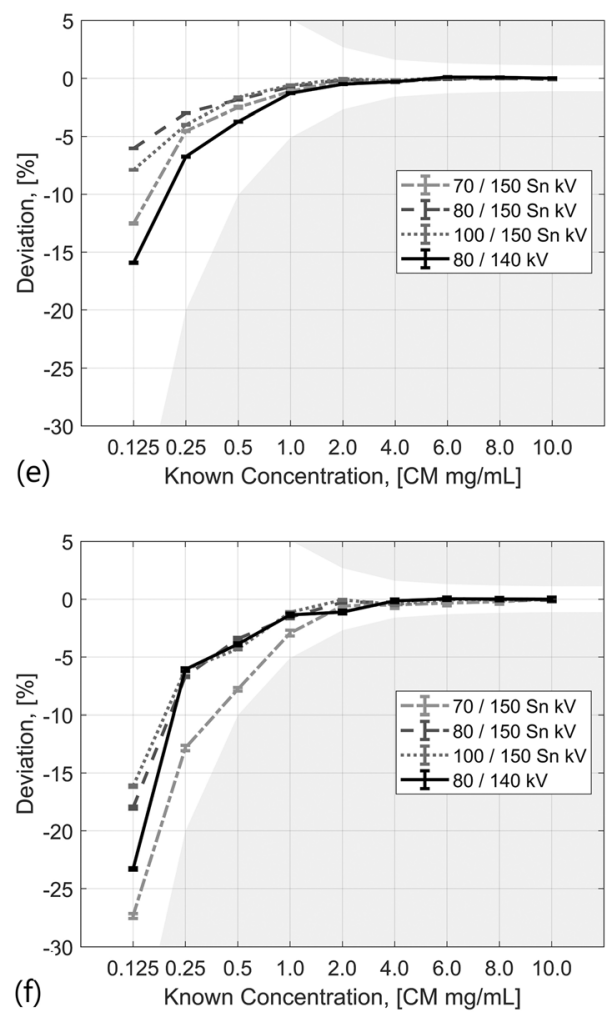

using automated exposure control (d). Relative deviations of the measured concentrations compared against the known concentrations for high dose measurements (e) and measurements using automated exposure control (f). The grey area depicts the area where the given tolerance is exceeded

energetic X-ray spectra $(70 \mathrm{kV}$ and $80 \mathrm{kV})$, the increased phantom size resulted in a shift of the mean X-ray spectra closer to the k-edge of holmium. The suddenly increased proportion of photoelectric absorption causes an increased total mass attenuation and therefore increased $\mathrm{CT}$ values. For higher energetic X-ray spectra (e.g., $140 \mathrm{kV}$ or $150 \mathrm{Sn} \mathrm{kV}$ ), the mean X-ray energy, as well as the spectral barycenter, moves away from the material's k-edge, leading to reduced mass attenuation and decreased CT values. This effect is different for materials such as iodinated $\mathrm{CM}$ (k-edge at $33 \mathrm{keV}$ )

Table 3 Linear fit parameters $(m$ for slope, $b$ for offset) and errors $\left(R^{2}\right.$ and root mean square error, RMSE) for the comparison of measured and known concentration with respect to different tube voltage combinations and tube currents

\begin{tabular}{|c|c|c|c|c|c|}
\hline \multirow[t]{2}{*}{ Tube current } & \multirow[t]{2}{*}{ Tube voltage combination } & \multicolumn{4}{|c|}{ Fit parameters and errors } \\
\hline & & $m$ & $b$ & $R^{2}$ & RMSE \\
\hline \multirow[t]{4}{*}{ CARE Dose 4D } & $70 / 150 \mathrm{Sn}$ & 1.03 & -0.32 & 0.99 & 0.25 \\
\hline & $80 / 150 \mathrm{Sn}$ & 1.01 & -0.16 & 1.00 & 0.12 \\
\hline & $100 / 150 \mathrm{Sn}$ & 1.02 & -0.16 & 1.00 & 0.12 \\
\hline & $80 / 140$ & 1.03 & -0.21 & 1.00 & 0.15 \\
\hline \multirow[t]{4}{*}{ High Dose } & $70 / 150 \mathrm{Sn}$ & 1.02 & -0.13 & 1.0 & 0.09 \\
\hline & $80 / 150 \mathrm{Sn}$ & 1.00 & -0.07 & 1.0 & 0.07 \\
\hline & $100 / 150 \mathrm{Sn}$ & 1.01 & -0.08 & 1.0 & 0.06 \\
\hline & $80 / 140$ & 1.02 & -0.17 & 1.0 & 0.12 \\
\hline
\end{tabular}



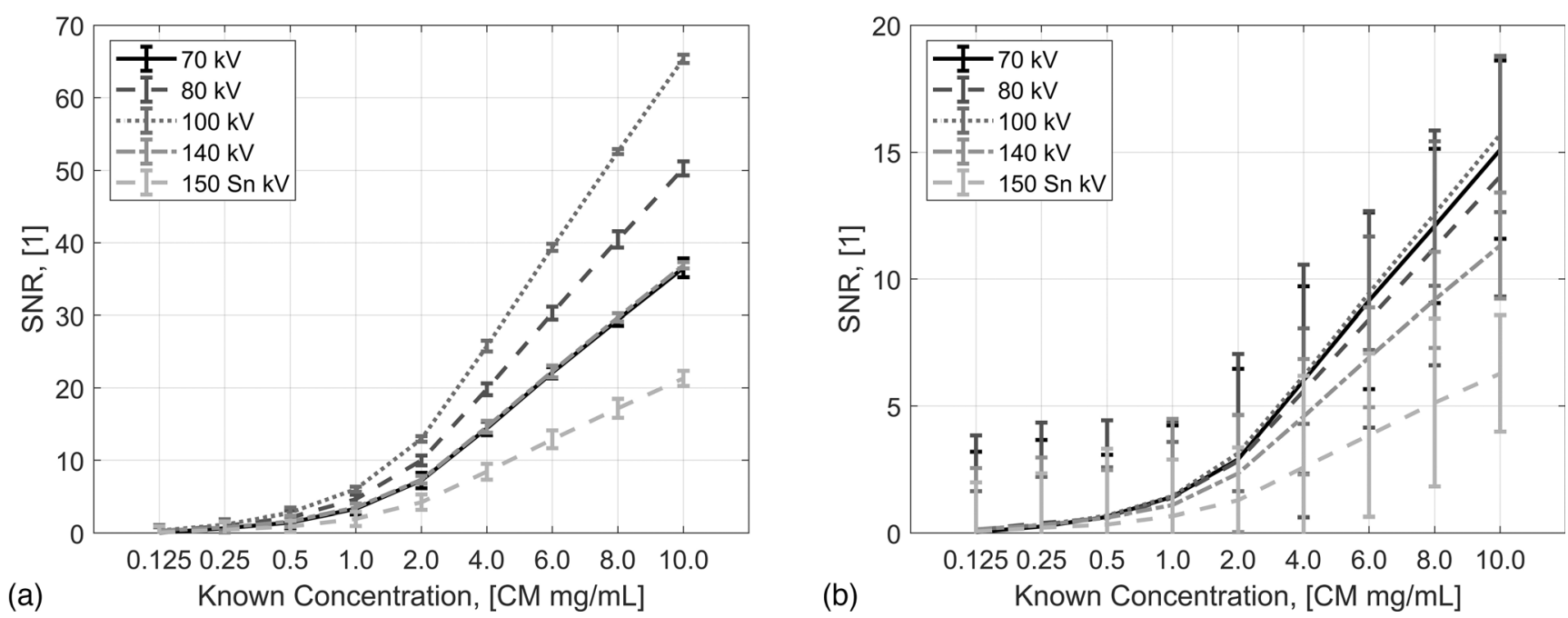

Fig. 4 Measured SNRs for all investigated ${ }^{165}$ HoMS solutions using high radiation dose (a) and typical clinical radiation dose (b)

because the mean energies of typical X-ray spectra are already beyond that energy.

Material quantification for concentrations down to 0.125 $\mathrm{mg} / \mathrm{mL}$ holmium was shown for all investigated tube voltage combinations and both evaluated dose levels. In the given experiment, a third-generation dual-source CT scanner was used. This scanner provides additional tube filtration to some tube voltages, which generally improves the spectral separation of dual-energy CT [6]. This option might not be available on all scanners, though. However, it was shown that a tube voltage combination of $80 / 140 \mathrm{kV}$ could also be used to achieve an acceptable quantification of holmium solutions.
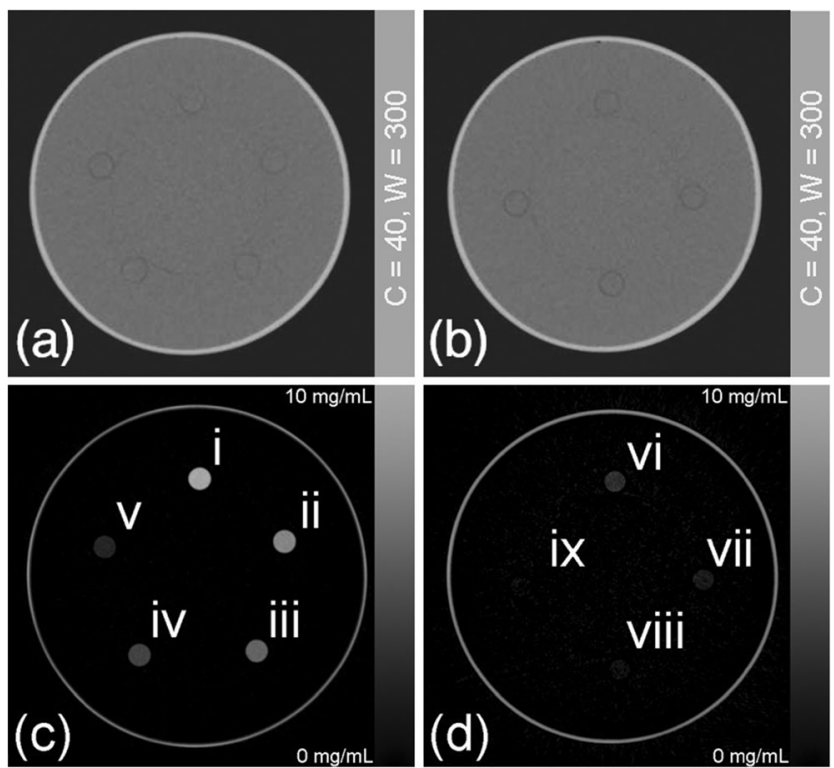

Fig. 5 a, b VNC images, i.e., images where the ${ }^{165} \mathrm{HoMS}$ is virtually removed. The material images $(\mathbf{c}, \mathbf{d})$ facilitate quantitative readout of the investigated ${ }^{165} \mathrm{HoMS}$ concentrations: 10.0, 8.0, 6.0, 4.0, and $2.0 \mathrm{mg}$ ${ }^{165} \mathrm{HoMS} / \mathrm{mL}$; vi-ix show the results for $1.0,0.5,0.25$, and $0.125 \mathrm{mg}$ ${ }^{165} \mathrm{HoMS} / \mathrm{mL}$
Measurements on three adult female NZW rabbits with subcutaneous VX-2 tumors, which were intratumorally injected with radioactive ${ }^{166} \mathrm{HoMS}$ and imaged post-mortem, demonstrated DECT-based holmium quantification in a scenario that is much closer to clinical reality. Using parameters identical to the calibration scans, the measured amounts of holmium corresponded well with the known injected amount of holmium with a maximum deviation of $11.7 \%$. Hypothetically, in a patient case where a tumor is injected with radioactive microspheres, the quantification is as follows: using the material decomposition method, the amount of holmium in mg per voxel can be calculated, with the known activity per mg at a certain timestamp given by the company in which the exact amount of activity at time-of-injection can be calculated. The last step is the conversion of an activity map (in $\mathrm{MBq}$ ) to a dose map (in Gy) which can be realized by using a Monte Carlo-based dose point kernel, which is available in dose evaluation software packages. The dose point kernel takes into account the different radiation types (energies and particles), tissue characteristics, and the volume in which the energy is absorbed. This allows for the evaluation of the absorbed dose distribution on the tumor and can be related to follow-up to tumor-response.

The observation of the characteristic mass attenuation behavior with regard to the photon's energies would facilitate kedge-sensitive imaging for either dual- or even multi-energetic applications. This could potentially be used for simultaneous imaging of a patient's vascularization and local accumulations of HoMS in tumorous regions within a single CT scan by virtually removing or quantifying one of the materials as shown in Figs. 5 and 6.

Impaired quality of the material separation that was found is largely a consequence of the experimental setup itself. The axial cross-sections of the investigated rabbits were rather elliptical, and the CT scanner did not correct for this shape with 

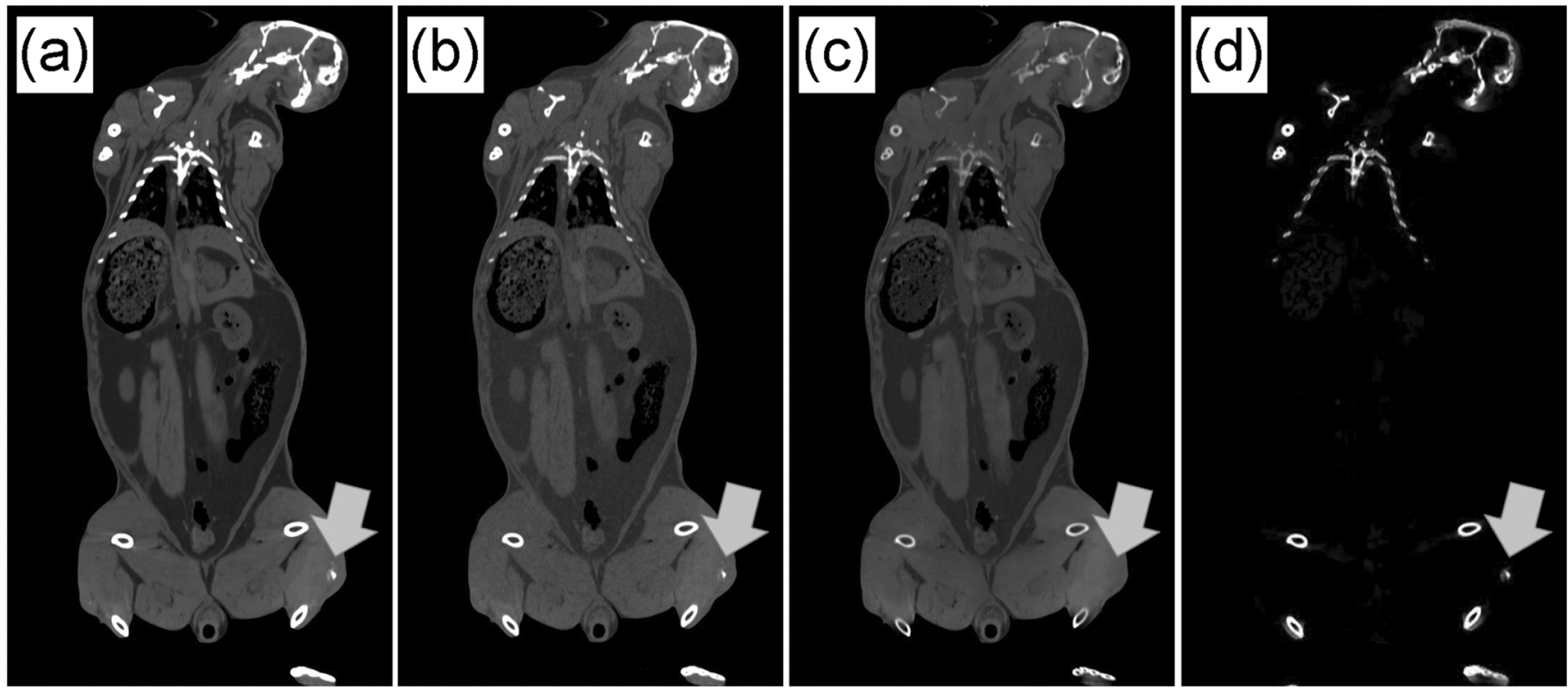

Fig. 6 Coronary slice of a scanned rabbit cadaver acquired with $80 \mathrm{kV}$ (a) and $150 \mathrm{Sn}(\mathrm{kV})$ (center $=150 \mathrm{HU}$, window $=600 \mathrm{HU})$. After applying A VNC image (c) as well as a material-specific image (d) was calculated

adaptive beam shaping. Additionally, the animals were partially frozen resulting in local changes of tissue densities leading to slightly deficiently determined soft tissue base material vectors. In our example, some tumor parts were not detected because of present partial volume effects as well as blooming artifacts caused by adjacent body materials (here: air and bone). In addition, the detection was suboptimal because third base materials are interpreted as a linear combination of the selected two base material vectors. This limitation could be overcome by extending the amount of applied or resolved Xray spectra and by adding a third material to the equation, as it can be done using alternative spectral $\mathrm{CT}$ technologies such as photon counting detectors (PCD) $[31,32]$. Investigating the quantification capability and robustness against clinically realistic artefacts that are per se inherent to a two material separation algorithm using a PCD-CT scanner is considered the next step in holmium microsphere imaging.

In conclusion, the first phantom and ex vivo holmium DECT data presented in this paper clearly show the feasibility to detect and quantify concentrations of holmium

Table 4 List of injected amounts of ${ }^{165} \mathrm{HoMS}$ and the detected amounts of ${ }^{165} \mathrm{HoMS}$ after material decomposition and quantification of the animal measurements. The relative deviations (with reference to the injected amounts) are shown in the fourth column

\begin{tabular}{llcc}
\hline Injections & $\begin{array}{l}\text { Injected amount of } \\
{ }^{165} \mathrm{HoMS}(\mathrm{mg})\end{array}$ & $\begin{array}{l}\text { Detected amount of } \\
165\end{array}$ & $\begin{array}{l}\text { Relative } \\
\text { deviation }(\%)\end{array}$ \\
\hline 1 & 5.61 & 6.3 & 11.4 \\
2 & 3.34 & 3.0 & -11.7 \\
3 & 5.96 & 5.5 & -7.7 \\
4 & 12.59 & 12.8 & 1.7 \\
\hline
\end{tabular}

microspheres. This could lead potentially to the increased clinical utility of DECT imaging for dose verification during and after holmium microspheres internal radiation therapy.

Acknowledgments The study was supported by Quirem Medical B.V. (Deventer, Netherlands) and Siemens Healthcare GmbH (Forchheim, Germany).

Funding information The study was supported by Quirem Medical B.V. (Deventer, Netherlands) and Siemens Healthcare GmbH (Forchheim, Germany).

\section{Compliance with ethical standards}

Guarantor The scientific guarantor of this publication is Frank Nijsen, Radboudumc; frank.nijsen@ radboudumc.nl.

Conflict of interest The authors of this manuscript declare relationships with the following companies:

Mr. Ralf Gutjahr and Dr. Bernhard Schmidt are employees of Siemens Healthcare GmbH. R.C. Bakker is funded by the Dutch Cancer Society research grant: 2014-7075 Mr. Feiko Tiessens is an employee of Quirem Medical B.V. J.F.W. Nijsen is the inventor on the patents on and related to the holmium microspheres which are assigned to University Medical Center Utrecht Holding B.V. and/or Quirem Medical (patent families: USA Patent No. 6,373,068 B1, PCT/NL03/00485, EP07112807.8, 10190254.2, P114198PC00, P112614NL00). He is co-founder and chief scientific officer of Quirem Medical and has a minority share in the company Quirem Medical. The activities of J. F.W. Nijsen within Quirem Medical are approved and supported by Dirkjan Masman (Director Technology Transfer Office Radboudumc) and Mathias Prokop (Head of the Department of Medical Imaging at Radboudumc). The Department of Radiology and Nuclear Medicine of the UMC Utrecht receives royalties from Quirem Medical B.V.

Statistics and biometry This is an observational/experimental study so this is not applicable.

No complex statistical methods were necessary for this paper. 
Informed consent Approval from the institutional animal care committee was obtained.

All experiments were performed in conduct with "The Netherlands Experiments on Animals Act (1977)" and "The European Convention for the Protection of Vertebrate Animals used for Experimental Purposes (Strasbourg, 18.III.1986)". Approval was obtained from the Utrecht University Animal Experiments Committee (DEC 2011.III.08.080).

Ethical approval Institutional Review Board approval was obtained.

All experiments were performed in conduct with "The Netherlands Experiments on Animals Act (1977)" and "The European Convention for the Protection of Vertebrate Animals used for Experimental Purposes (Strasbourg, 18.III.1986)". Approval was obtained from the Utrecht University Animal Experiments Committee (DEC 2011.III.08.080).

\section{Methodology}

- observational/experimental

Open Access This article is licensed under a Creative Commons Attribution 4.0 International License, which permits use, sharing, adaptation, distribution and reproduction in any medium or format, as long as you give appropriate credit to the original author(s) and the source, provide a link to the Creative Commons licence, and indicate if changes were made. The images or other third party material in this article are included in the article's Creative Commons licence, unless indicated otherwise in a credit line to the material. If material is not included in the article's Creative Commons licence and your intended use is not permitted by statutory regulation or exceeds the permitted use, you will need to obtain permission directly from the copyright holder. To view a copy of this licence, visit http://creativecommons.org/licenses/by/4.0/.

\section{References}

1. Di Chiro G, Brooks RA, Kessler RM et al (1979) Tissue signatures with dual-energy computed tomography. Radiology 131:521-523

2. Genant HK, Boyd D (1977) Quantitative bone mineral analysis using dual energy computed tomography. Invest Radiol 12:54555

3. McDavid WD, Waggener RG, Dennis MJ, Sank VJ, Payne WH (1977) Estimation of chemical composition and density from computed tomography carried out at a number of energies. Invest Radiol 12:189-194

4. Millner MR, McDavid WD, Waggener RG, Dennis MJ, Payne WH, Sank VJ (1979) Extraction of information from CT scans at different energies. Med Phys 6:70-71

5. Rutherford RA, Pullan BR, Isherwood I (1976) Measurement of effective atomic number and electron density using an EMI scanner. Neuroradiology 11:15-21

6. Primak AN, Giraldo JCR, Eusemann CD et al (2010) Dual-source dual-energy CT with additional tin filtration: dose and image quality evaluation in phantoms and in-vivo. AJR Am J Roentgenol 195: 1164

7. Kelcz F, Joseph PM, Hilal SK (1979) Noise considerations in dual energy CT scanning. Med Phys 6:418-425

8. Johnson TRC, Krauss B, Sedlmair M et al (2007) Material differentiation by dual energy CT: initial experience. Eur Radiol 17: $1510-1517$

9. Pelgrim GJ, van Hamersvelt RW, Willemink MJ et al (2017) Accuracy of iodine quantification using dual energy CT in latest generation dual source and dual layer CT. Eur Radiol 27:39043912
10. Reimann AJ, Rinck D, Birinci-Aydogan A et al (2007) Dual-source computed tomography: Advances of improved temporal resolution in coronary plaque imaging. Invest Radiol 42:196-203

11. Yeh BM, Shepherd JA, Wang ZJ, Hui ST, Hartman RP, Prevrhal S (2009) Dual-energy and low-kVp CT in the abdomen. Am J Roentgenol:47-54

12. Alvarez RE, Macovski A (1976) Energy-selective reconstructions in X-ray computerised tomography. Phys Med Biol 21:733

13. Coursey CA, Nelson RC, Boll DT et al (2010) Dual-energy multidetector CT: how does it work, what can it tell us, and when can we use it in abdominopelvic imaging? Radiographics 30:1037-1055

14. Yang C-B, Zhang S, Jia Y-J et al (2017) Dual energy spectral CT imaging for the evaluation of small hepatocellular carcinoma microvascular invasion. Eur J Radiol 95:222-227

15. Li Y, Shi G, Wang S, Wang S, Wu R (2013) Iodine quantification with dual-energy CT: phantom study and preliminary experience with VX2 residual tumour in rabbits after radiofrequency ablation. Br J Radiol 86:20130143

16. Primak AN, Fletcher JG, Vrtiska TJ et al (2007) Noninvasive differentiation of uric acid versus non-uric acid kidney stones using dual-energy CT. Acad Radiol 14:1441-1447

17. Graser A, Johnson TRC, Bader M et al (2008) Dual energy CT characterization of urinary calculi: initial in vitro and clinical experience. Invest Radiol 43:112-119

18. Fukuda T, Umezawa Y, Asahina A, Nakagawa H, Furuya K, Fukuda K (2017) Dual energy CT iodine map for delineating inflammation of inflammatory arthritis. Eur Radiol 27:5034-5040

19. Bongartz T, Glazebrook KN, Kavros SJ et al (2015) Dual-energy CT for the diagnosis of gout: an accuracy and diagnostic yield study. Ann Rheum Dis 74:1072-1077

20. Smits MLJ, Elschot M, van den Bosch MAAJ et al (2013) In Vivo Dosimetry Based on SPECT and MR Imaging of 166HoMicrospheres for Treatment of Liver Malignancies. J Nucl Med 54:2093-2100

21. van Nimwegen SA, Bakker RC, Kirpensteijn J et al (2017) Intratumoral injection of radioactive holmium (166 Ho) microspheres for treatment of oral squamous cell carcinoma in cats. Vet Comp Oncol 16:114-124

22. Bakker RC, van Es RJJ, Rosenberg AJWP et al (2018) Intratumoral injection of radioactive holmium-166 microspheres in recurrent head and neck squamous cell carcinoma. Nucl Med Commun. https://doi.org/10.1097/mnm.0000000000000792:1

23. Nijsen JFW, Cornelis Krijger G, van het Schip A (2007) The bright future of radionuclides for cancer therapy. Anticancer Agents Med Chem 7:271-290

24. Seevinck PR, Seppenwoolde J-H, de Wit TC et al (2007) Factors affecting the sensitivity and detection limits of MRI, CT, and SPECT for multimodal diagnostic and therapeutic agents. Anticancer Agents Med Chem 7:317-334

25. Seppenwoolde J-H, Nijsen JFW, Bartels LW, Zielhuis SW, van het Schip AD, Bakker CJG (2005) Internal radiation therapy of liver tumors: Qualitative and quantitative magnetic resonance imaging of the biodistribution of holmium-loaded microspheres in animal models. Magn Reson Med 53:76-84

26. van de Maat GH, Seevinck PR, Elschot M et al (2013) MRI-based biodistribution assessment of holmium-166 poly(L-lactic acid) microspheres after radioembolisation. Eur Radiol 23:827-835

27. Stierstorfer K, Rauscher A, Boese J, Bruder H, Schaller S, Flohr T (2004) Weighted FBP — a simple approximate 3D FBP algorithm for multislice spiral CT with good dose usage for arbitrary pitch. Phys Med Biol 49:2209

28. Nijsen JFW, Seppenwoolde J-H, Havenith T, Bos C, Bakker CJG, van het Schip AD (2004) Liver tumors: MR imaging of radioactive holmium microspheres - phantom and rabbit study. Radiology 231:491-499 
29. Liu X, Yu L, Primak AN, McCollough CH (2009) Quantitative imaging of element composition and mass fraction using dualenergy CT: three-material decomposition. Med Phys 36:1602-1609

30. Siemens Healthineers Global (2017) SyngoCT Postprocessing applications - Instructions For Use - syngoCT Workplace syngo CT VB20

31. Symons R, Cork TE, Lakshmanan MN et al (2017) Dual-contrast agent photon-counting computed tomography of the heart: initial experience. Int J Cardiovasc Imaging 33:1253-1261
32. Faby S, Maier J, Sawall S et al (2016) An efficient computational approach to model statistical correlations in photon counting x-ray detectors. Med Phys 43:3645-3960

Publisher's note Springer Nature remains neutral with regard to jurisdictional claims in published maps and institutional affiliations. 